\title{
Esophageal foreign-body impactions: epidemiology, time trends, and the impact of the increasing prevalence of eosinophilic esophagitis
}

\author{
Sarah L.W. Sperry, BA ${ }^{1,2}$, Seth D. Crockett, MD, MPH $^{1,2}$, C. Brock Miller, MD ${ }^{1}$, Nicholas J. \\ Shaheen, MD, MPH ${ }^{1,2}$, and Evan S. Dellon, MD, MPH ${ }^{1,2}$ \\ ${ }^{1}$ Center for Gastrointestinal Biology and Disease, Department of Medicine, University of North \\ Carolina School of Medicine, Chapel Hill, North Carolina, USA \\ 2Division of Gastroenterology and Hepatology, Department of Medicine, University of North \\ Carolina School of Medicine, Chapel Hill, North Carolina, USA
}

\section{Abstract}

Background-The epidemiology of esophageal foreign-body impaction (EFBI) is poorly described, and the impact of the increasing prevalence of eosinophilic esophagitis (EoE) on this is unknown.

Objective-To assess the characteristics of patients with EFBI, to determine whether EFBI cases increased in proportion to EoE cases, and to identify predictors of EFBI.

Design-Retrospective study.

Setting-Tertiary care center.

Patients-Cases of EFBI from 2002 to 2009 were identified by querying billing, clinical, and endoscopy databases for the International Classification of Diseases, 9th Revision, Clinical Modification code 935.1, "foreign body in the esophagus." Charts were reviewed to confirm EFBI and to extract pertinent data. Cases of EoE were defined per guidelines.

Results-Of 548 patients with EFBI (59\% male, 68\% white, bimodal age distribution), 482 $(88 \%)$ required a procedure, 347 (63\%) had food impactions, and $51(9 \%)$ had EoE. EFBIs increased over the study time frame, and the number of EGDs performed for EFBI nearly quadrupled. Increasing diagnosis of EoE did not fully account for this trend, but only $27 \%$ of patients who underwent EGD had esophageal biopsies. Of patients who underwent biopsy, $46 \%$ had EoE. EoE was the strongest predictor of multiple EFBIs (odds ratio 3.5; 95\% CI, 1.8-7.0).

Limitations-Retrospective, single-center study.

Conclusions-The number of EGDs performed for EFBI has increased dramatically at our center, but increasing EoE prevalence only partially explains this trend. Because only a minority of EFBI patients underwent biopsies and because nearly half of those who did undergo biopsy had

Copyright (C) 2011 by the American Society for Gastrointestinal Endoscopy

Reprint requests: Evan S. Dellon, MD, CB\#7080, Bioinformatics Building, 130 Mason Farm Road, UNC-CH, Chapel Hill, NC 27599-7080.

Current affiliations: Center for Esophageal Diseases and Swallowing, Department of Medicine, University of North Carolina School of Medicine, Chapel Hill, North Carolina, USA.

If you would like to chat with an author of this article, you may contact Dr Dellon at edellon@ med.unc.edu.

DISCLOSURE: The authors disclosed no financial relationships relevant to this publication. 
EoE, the incidence of EoE may be substantially underestimated. Physician education is needed to increase the proportion of subjects with EFBI who undergo biopsies.

Esophageal foreign-body impaction (EFBI) is a GI emergency, often requiring presentation to an emergency department (ED) for urgent evaluation and treatment. ${ }^{1}$ The etiologies are numerous and include inadvertent swallowing of coins in children, complications of reflux disease such as peptic strictures, Schatzki's rings, motility disorders such as achalasia, and malignancy. ${ }^{1-3}$

In recent years, EFBI has also been recognized as a major presenting feature of eosinophilic esophagitis (EoE), particularly in adults. Several studies suggest that EoE is now the leading cause of food impaction in patients presenting to an $\mathrm{ED}$, accounting for more than $50 \%$ of episodes. ${ }^{4,5}$ The incidence and prevalence of EoE have increased significantly over the past decade in both children and adults, ${ }^{6-10}$ but the impact of EoE on the epidemiology of EFBI is not well understood.

The purposes of this study were to assess the characteristics of patients presenting to a tertiary care center with EFBI, to determine whether the number of EFBI cases has increased with the increasing prevalence of EoE, and to identify predictors of EFBI. We hypothesized that the incidence of EFBI increased in proportion to the incidence of EoE and that a substantial proportion of EFBI would occur in association with EoE.

\section{METHODS}

We conducted a retrospective study of all patients presenting with EFBI to University of North Carolina (UNC) Hospital from June 2002 through December 2009. All data were collected from the single UNC Hospital location in Chapel Hill, North Carolina. To improve the sensitivity of our case-finding strategy, potential cases of EFBI were identified by querying 3 separate electronic databases for all records with the International Classification of Diseases, 9th Revision, Clinical Modification (ICD-9-CM) code 935.1, "foreign body in the esophagus." The 3 sources were (1) the UNC Hospital billing database (available 20022009), (2) the UNC comprehensive clinical data warehouse (available 2006-2009), and (3) the UNC endoscopy database (2002-2009; Provation Md, Wolters-Kluwer, Minneapolis, Minn). This ICD-9 code was constant over the study time frame, and there were no changes in billing practice or the endoscopy reporting system during this time period.

Charts were then reviewed to confirm EFBI status, defined as ingestion of food or a potentially obstructing foreign body, presentation with symptoms of esophageal bolus impaction (eg, acute dysphagia, chest pain, foreign-body sensation, inability to control secretions), and one of the following: either a procedure (ie, upper endoscopy or rigid esophagoscopy) that demonstrated bolus impaction, a response to medical therapy (eg, glucagon) that resulted in witnessed clearing of the obstructing bolus either by vomiting or swallowing, or a witnessed resolution of the impaction in the ED before undergoing a procedure. Patients were excluded if the ICD-9-CM code 935.1 could not be linked to an acute care visit or a procedure with the features listed. Pertinent data from first-time EFBI cases were extracted and included date of EFBI, age, sex, race, impacted item, procedure(s) performed, procedure complications (unsuccessful endoscopy, respiratory compromise, cardiac arrhythmia, hypotension, mucosal injury, perforation, death), endoscopic or surgical techniques used to clear the impacted bolus, esophageal biopsy sample procurement (if performed), esophageal dilation (if performed), GI comorbidities, and previous or subsequent episodes of EFBI. Patients with more than 1 occurrence of EFBI during this time period, either at our institution or reported by history, were categorized as having recurrent 
EFBI. Cases of GERD were defined by the diagnosis in the medical record. Cases of EoE were defined per 2007 consensus guidelines. ${ }^{10}$

Specifically, subjects had clinical symptoms of esophageal dysfunction, 15 or more eosinophils in at least 1 high-power field (eos/hpf), and either a lack of histologic response to 6 to 8 weeks of treatment with a high-dose proton pump inhibitor (PPI), or a normal pH monitoring study of the distal esophagus. Cases of EoE were reconfirmed by crossreferencing our EoE patient database, which is a comprehensive registry of all EoE patients seen at UNC from 2000 to the present.

Descriptive statistics were used to summarize the findings. Because of the heterogeneity of presentation with EFBI, bivariate analyses were performed comparing food with nonfood impactions, EoE patients with non-EoE patients, and recurrent impactions with a single impaction. Means were compared with $t$ test and proportions were compared with the $\chi^{2}$ test. For variables where there were not normal distributions, medians were compared by using the Wilcoxon rank-sum test. Multivariate analysis assessing predictors of recurrent EFBI was performed with logistic regression. Potential confounding factors were identified on bivariate analysis and included in the initial model. A backward elimination strategy was used to reduce the model, retaining those covariates whose removal caused a change in estimate greater than $10 \%$. The final model included age, sex, race, GERD, EoE, and whether a procedure was performed. This study was approved by the UNC Institutional Review Board.

\section{RESULTS}

\section{Patient characteristics and time trends}

Overall, 548 patients with EFBI were identified from our search (Table 1). Subjects were $59 \%$ male and $68 \%$ white and ranged in age from 3 months to 99 years. Age distribution was bimodal, with peaks between 1 to 5 years and 40 to 80 years; the median age was 39 years. GI comorbidities contributing to the episode of EFBI included GERD (10\%), esophageal stricture (12\%), Schatzki's ring (7\%), achalasia (5\%), malignancy (2\%), and EoE (9\%); 264 patients (48\%) had no known GI-related diagnosis. The most common type of impacted item was nonmeat food, occurring in $31 \%$ of patients, followed by meat in $25 \%$ patients. Coins were the impacted item in $21 \%$ of patients, and other items included plastic toys (eg, Lego or Light Bright pieces), beads, batteries, and a golf tee (Table 1).

Over the 8-year time frame of this study, the annual incidence of EFBI increased from 15 episodes in 2002 to 100 in 2009 (Fig. 1A). The proportion of EFBI that was caused by food impaction similarly increased (12 episodes in 2002 to 51 in 2009). The increasing incidence was seen in both children and adults, but appears to have plateaued in the most recent 2 to 3 years of the study.

\section{Procedure characteristics}

A total of 482 patients $(88 \%)$ required a procedure to treat their EFBI (Table 2). The majority of patients $(363,75 \%)$ underwent a flexible upper endoscopy, whereas $126(26 \%)$ patients underwent an esophagoscopy or laryngoscopy performed by colleagues in the otolaryngology department. One patient underwent surgical exploration for a mediastinal mass that extended into the esophagus. Over the study time frame, the total number of EGDs performed for EFBI nearly quadrupled (Fig. 1B). The most common techniques used to clear the bolus impaction included extraction (49\%), with tools such as rat-tooth or other nonbiopsy forceps, Roth nets, snares, coin graspers, and gentle advancement of the impacted bolus (typically food) into the stomach with an endoscope (45\%). Additionally, 66 of the 
patients (14\%) underwent esophageal dilation during the initial procedure after bolus extraction for treatment of esophageal strictures or rings. A through-the-scope balloon dilator was used in 37 of these patients (56\%), a Savary dilator in 28 (42\%), and a Maloney dilator in 1 patient.

Only a small minority of patients (3\%) experienced a procedure-related complication. The most common complication was an unsuccessful EGD, resulting in subsequent treatment in the operating room from the ear, nose, and throat (ENT) or GI surgical service, which occurred in 7 patients (Table 2). Two patients had bronchospasm, 1 of whom had aspiration; 1 patient had hemorrhage (not requiring administration of blood products); 3 had superficial mucosal tears; and 1 patient went into atrial fibrillation during the procedure. None of these complications occurred in patients who underwent an esophageal biopsy during the procedure.

\section{Type of impactions}

When comparing food and nonfood impactions, we observed several significant associations (Table 3 ). There was a significant difference in mean age (50 years vs 14 years) between food impactions and nonfood impactions $(P<.0001)$ as well as race $(77 \%$ nonwhite vs $53 \%$ white, $P<.001)$. GERD and other GI-related diagnoses were also associated with food impactions, whereas the vast majority of nonfood impactions were related to swallowed coins in children presumed not to have underlying esophageal pathology. However, patients who had food impactions were more likely to undergo esophageal biopsy than those with nonfood impactions ( $23 \%$ vs $8 \%, P<.001)$.

\section{EoE and EFBI}

Fifty-one patients (9\%) with EFBI had a diagnosis of EoE by consensus guidelines, with 45 of those patients receiving a new diagnosis after the episode of EFBI. When limited to cases of esophageal food impactions, 42 patients (12\%) had EoE. There were 9 EoE patients with nonfood impactions ( 1 swallowed a coin and 1 swallowed a tooth; in the other 7 patients, the type of nonfood-impacted object was not specified). There were an additional 7 patients who had 15 or more eos/hpf on a biopsy sample, but who did not meet our definition of EoE because GERD had not been excluded. Between 2002 and 2009, the number of EoE patients undergoing EGD for EFBI increased substantially, but did not completely account for the overall increase in the number of EGDs or EFBIs (Fig. 1B). However, only 98 of the 363 patients $(27 \%)$ who underwent EGD underwent an esophageal biopsy during the initial procedure related to their foreign-body impaction, so not all cases of EoE may have been identified. For example, the $45 \mathrm{EoE}$ patients who received a diagnosis after presenting with EFBI comprised a substantial proportion (46\%) of patients who underwent esophageal biopsy on upper endoscopy. There was an increase in the proportion of cases in which biopsies were performed, from less than 5\% in 2002 to 2004 to greater than $20 \%$ after 2005, but the overall proportion was still less than $30 \%$. Of note, all biopsies were performed during EGDs performed by a GI endoscopist; none were performed during procedures performed by the ENT service.

There were multiple differences between patients with EFBI and EoE and those without EoE (Table 4). Compared with patients without known EoE, EoE was associated with younger age (26 years vs 39 years, $P=.005)$, male sex ( $82 \%$ vs $18 \%, P<.001)$, and white race (92\% vs $66 \%, P<.001)$. Food impaction was significantly more likely to occur in patients with EoE compared with those without ( $82 \%$ vs $61 \%, P=.007$ ), but there was no significant association with type of food. Multiple episodes of EFBI occurred more frequently in patients with EoE compared with those without EoE ( $35 \%$ vs 17\%, $P=.002$ ). 


\section{Predictors of recurrent EFBI episodes}

A total of 103 patients (19\%) were categorized as having recurrent episodes of EFBI. On bivariate analysis, multiple episodes of EFBI were associated with older age, male sex, food as the cause of the impacted bolus, a diagnosis of GERD, and a diagnosis of EoE (Table 5). On multivariate logistic regression analysis, after adjusting for age, sex, race, GERD, and whether a procedure was required, EoE was the strongest independent predictor of multiple EFBIs (odds ratio 3.5; 95\% CI, 1.8-7.0).

\section{DISCUSSION}

EFBI is a GI emergency that frequently requires urgent evaluation and procedural intervention. Although numerous etiologies have been documented in the literature, ${ }^{1-3}$ the effect of EoE on the epidemiology of EFBI is less well understood. Because the incidence and prevalence of EoE are known to be increasing, ${ }^{6-8}$ the goal of this study was to characterize a large number of EFBI cases and assess the impact of EoE on EFBI.

Over the time frame of this study, we observed a striking increase in the number of EFBIs that occurred each year, as well as a concomitant increase in the number of EGDs performed, although this increase appeared to plateau in the last years of the study. The reasons for the increasing number of EFBI cases are partially explained by an increase in the number of EoE cases over the same time frame, but this alone does not explain the dramatic increase in annual EFBIs. However, the low overall rate of biopsy sample procurement during EGD, which remained less than $30 \%$ despite increasing over the study time frame, implies that our findings likely underestimate the incidence of EoE in the population that presents with EFBI. In addition, we found that EoE was the strongest predictor of recurrent episodes of EFBI.

Food impaction in EoE is common, with frequencies ranging from $25 \%$ to $100 \%$ in previous studies, depending on the specific population. ${ }^{9-15}$ In 2 previous prospective studies specifically examining the prevalence of EoE in patients presenting with food impaction, ${ }^{4,5}$ at least 50\% of patients had EoE diagnosed. Among our patients who presented with esophageal food impactions, $12 \%$ had EoE, a noticeably lower proportion. The reasons for this are likely twofold. First, as previously mentioned, less than one third of patients in this study underwent esophageal biopsies either during the food impaction or during a follow-up endoscopy, so EoE may have been missed. When examining just the patients who underwent biopsies, the proportion of EoE patients was approximately $50 \%$, in line with the previous studies. Second, the previous studies used a histologic definition of EoE based on esophageal eosinophilia at the time of food impaction, regardless of PPI status. In our study, the EoE patients all had a follow-up endoscopy with biopsies demonstrating at least 15 eos/ hpf while on twice-daily PPI therapy, consistent with consensus guidelines ${ }^{10}$; there were an additional 7 patients who had esophageal eosinophilia but in whom EoE could not be confirmed. Recent data suggest that a high proportion of patients with esophageal eosinophilia may have normalization of their biopsy samples with PPI use, ${ }^{16-18}$ and this may be particularly true when biopsy samples are only obtained when there is acute tissue injury, as is often the case in EFBIs. ${ }^{19}$ In the study by Desai et al, ${ }^{4}$ whereas 17 of 31 patients with food impaction met the criteria for EoE ( $\geq 20$ eos/hpf), only 8 were PPI nonresponders who were then treated with topical steroids. The implication of this is that a substantial proportion of patients who present with EFBI will have biopsy findings with esophageal eosinophilia suspicious for EoE, but will have resolution of eosinophilia on PPI therapy. Interestingly, a recent retrospective study of EFBI in children found that of the 72 food impaction events, biopsy samples were only obtained in $49 \% .{ }^{20}$ However, in that group, biopsy sample findings were abnormal and with some degree of esophageal eosinophilia in $88 \%$. Perhaps efforts made to educate physicians about the incidence of EoE among patients 
who present with EFBI will help increase the biopsy rate during EGD and allow for a more accurate assessment of the prevalence of EoE in cases of EFBI.

It is important to acknowledge the strengths and limitations of this study. First, we performed an exhaustive review of multiple electronic resources during the study period to capture all episodes of EFBI seen at our institution. Therefore, we have a large sample size with the ability to visualize time trends and analyze predictors. However, because this was a retrospective study, we could not obtain data from patients who were seen in our system once for the acute event and then had follow-up care elsewhere. Consequently, the rate of recurrent EFBI may be underestimated. We attempted to counter this potential weakness by extensively characterizing each patient and EFBI case, and our data demonstrate demographic and disease-specific findings similar to those of other published reports of EFBI. ${ }^{1,4,5,20-23}$ Although this yielded many study variables, some of which were heterogeneous, we focused our analysis to be in line with the aims of the study. In addition, we adhered to the published guidelines for diagnosis of $\mathrm{EoE}^{10}$ and further cross-referenced these patients with our EoE patient database to confirm their status. Given that this was a retrospective study, we were not able to apply a similar set of criteria to the diagnosis of GERD, which was instead based on diagnosis in the patient's medical record and thus may be subject to error.

In summary, the number of EGDs performed for EFBI has increased dramatically at our center, and the increasing prevalence of EoE in this patient population only partially explains this trend. However, because only a minority of patients underwent biopsies at the time of EFBI, the incidence of EoE in this population is likely substantially underestimated, as supported by our finding that the large proportion of patients who did undergo a biopsy were found to have EoE. The complication rate during an EGD performed for EFBI is quite low in our data, and no complications occurred in patients who had esophageal biopsy samples taken. Therefore, it is important to obtain esophageal biopsy samples in all patients with EFBI without a readily apparent structural cause to assess for EoE as the underlying etiology. It also may be necessary to repeat an EGD subsequently after the patient has been on a PPI to confirm a diagnosis of EoE, assess tissue healing, or perform dilation. Physician education regarding the high prevalence of EoE in subjects with food impaction is likely required to change practice and increase the proportion of subjects with EFBI who undergo biopsy.

\section{Acknowledgments}

This work was supported by a grant from the Doris Duke Charitable Foundation to the University of North Carolina-Chapel Hill (S.L.W.S.), NIH training grant T32DK007634 (S.D.C.), NIH award number KL2RR025746 from the National Center for Research Resources (E.S.D.), and a Junior Faculty Development Award from the American College of Gastroenterology (E.S.D.).

\section{Abbreviations}

$\begin{array}{ll}\text { ED } & \text { emergency department } \\ \text { EFBI } & \text { esophageal foreign-body impaction } \\ \text { ENT } & \text { ear, nose, and throat } \\ \text { EoE } & \text { eosinophilic esophagitis } \\ \text { eos/hpf } & \text { eosinophils per high-power field } \\ \text { ICD-9-CM } & \text { International Classification of Diseases, 9th Revision, Clinical Modification }\end{array}$



PPI
proton pump inhibitor
UNC
University of North Carolina

\section{REFERENCES}

1. Longstreth GF, Longstreth KJ, Yao JF. Esophageal food impaction: epidemiology and therapy. A retrospective, observational study. Gastrointest Endosc. 2001; 53:193-198. [PubMed: 11174291]

2. Vicari JJ, Johanson JF, Frakes JT. Outcomes of acute esophageal food impaction: success of the push technique. Gastrointest Endosc. 2001; 53:178-181. [PubMed: 11174288]

3. Weinstock LB, Shatz BA, Thyssen SE. Esophageal food bolus obstruction: evaluation of extraction and modified push techniques in 75 cases. Endoscopy. 1999; 31:421-425. [PubMed: 10494678]

4. Desai TK, Stecevic V, Chang CH, et al. Association of eosinophilic inflammation with esophageal food impaction in adults. Gastrointest Endosc. 2005; 61:795-801. [PubMed: 15933677]

5. Kerlin P, Jones D, Remedios M, et al. Prevalence of eosinophilic esophagitis in adults with food bolus obstruction of the esophagus. J Clin Gastroenterol. 2007; 41:356-361. [PubMed: 17413601]

6. Noel RJ, Putnam PE, Rothenberg ME. Eosinophilic esophagitis. N Engl J Med. 2004; 351:940-941. [PubMed: 15329438]

7. Straumann A, Simon HU. Eosinophilic esophagitis: escalating epidemiology? J Allergy Clin Immunol. 2005; 115:418-419. [PubMed: 15696105]

8. Prasad GA, Alexander JA, Schleck CD, et al. Epidemiology of eosinophilic esophagitis over three decades in Olmsted County, Minnesota. Clin Gastroenterol Hepatol. 2009; 7:1055-1061. [PubMed: 19577011]

9. Dellon ES, Gibbs WB, Fritchie KJ, et al. Clinical, endoscopic, and histologic findings distinguish eosinophilic esophagitis from gastroesophageal reflux disease. Clin Gastroenterol Hepatol. 2009; 7:1305-1313. quiz 1261. [PubMed: 19733260]

10. Furuta GT, Liacouras CA, Collins MH, et al. Eosinophilic esophagitis in children and adults: a systematic review and consensus recommendations for diagnosis and treatment. Sponsored by the American Gastroenterological Association (AGA) Institute and North American Society of Pediatric Gastroenterology, Hepatology, and Nutrition. Gastroenterology. 2007; 133:1342-1363. [PubMed: 17919504]

11. Dellon ES, Aderoju A, Woosley JT, et al. Variability in diagnostic criteria for eosinophilic esophagitis: a systematic review. Am J Gastroenterol. 2007; 102:2300-2313. [PubMed: 17617209]

12. Prasad GA, Talley NJ, Romero Y, et al. Prevalence and predictive factors of eosinophilic esophagitis in patients presenting with dysphagia: a prospective study. Am J Gastroenterol. 2007; 102:2627-2632. [PubMed: 17764492]

13. Veerappan GR, Perry JL, Duncan TJ, et al. Prevalence of eosinophilic esophagitis in an adult population undergoing upper endoscopy: a prospective study. Clin Gastroenterol Hepatol. 2009; 7:420-426. 426.e1-2. [PubMed: 19162236]

14. Mackenzie SH, Go M, Chadwick B, et al. Eosinophilic oesophagitis in patients presenting with dysphagia-a prospective analysis. Aliment Pharmacol Ther. 2008; 28:1140-1146. [PubMed: 18624788]

15. Straumann A, Bussmann C, Zuber M, et al. Eosinophilic esophagitis: analysis of food impaction and perforation in 251 adolescent and adult patients. Clin Gastroenterol Hepatol. 2008; 6:598-600. [PubMed: 18407800]

16. Molina-Infante J, Ferrando-Lamana L, Ripoll C, et al. Esophageal eosinophilic infiltration responds to proton pump inhibition in most adults. Clin Gastroenterol Hepatol. 2011; 9:110-117. [PubMed: 20920599]

17. Sayej WN, Patel R, Baker RD, et al. Treatment with high-dose proton pump inhibitors helps distinguish eosinophilic esophagitis from noneosinophilic esophagitis. J Pediatr Gastroenterol Nutr. 2009; 49:393-399. [PubMed: 19633574] 
18. Spechler SJ, Genta RM, Souza RF. Thoughts on the complex relationship between gastroesophageal reflux disease and eosinophilic esophagitis. Am J Gastroenterol. 2007; 102:1301-1306. [PubMed: 17531015]

19. Heller JC, Freeman S, Furuta GT. Curious elements of esophageal foreign body impaction and eosinophilic esophagitis. Gastroenterol Hepatol (N Y). 2009; 5:836-838. [PubMed: 20567528]

20. Hurtado CW, Furuta GT, Kramer RE. Etiology of esophageal food impactions in children. J Pediatr Gastroenterol Nutr. 2011; 52:43-46. [PubMed: 20975581]

21. Byrne KR, Panagiotakis PH, Hilden K, et al. Retrospective analysis of esophageal food impaction: differences in etiology by age and gender. Dig Dis Sci. 2007; 52:717-721. [PubMed: 17253139]

22. Nadir A, Sahin E, Nadir I, et al. Esophageal foreign bodies: 177 cases. Dis Esophagus. 2011; 24:69. [PubMed: 20626451]

23. Wu WT, Chiu CT, Kuo CJ, et al. Endoscopic management of suspected esophageal foreign body in adults. Dis Esophagus. 2011; 24:131-137. [PubMed: 20946132] 


\section{Take-home Message}

- Because only a minority of patients with esophageal foreign-body impaction (EFBI) underwent biopsies and because nearly half of those who did had eosinophilic esophagitis (EoE), the incidence of EoE may be substantially underestimated.

- Physician education is needed to increase the proportion of subjects with EFBI who undergo biopsies. 

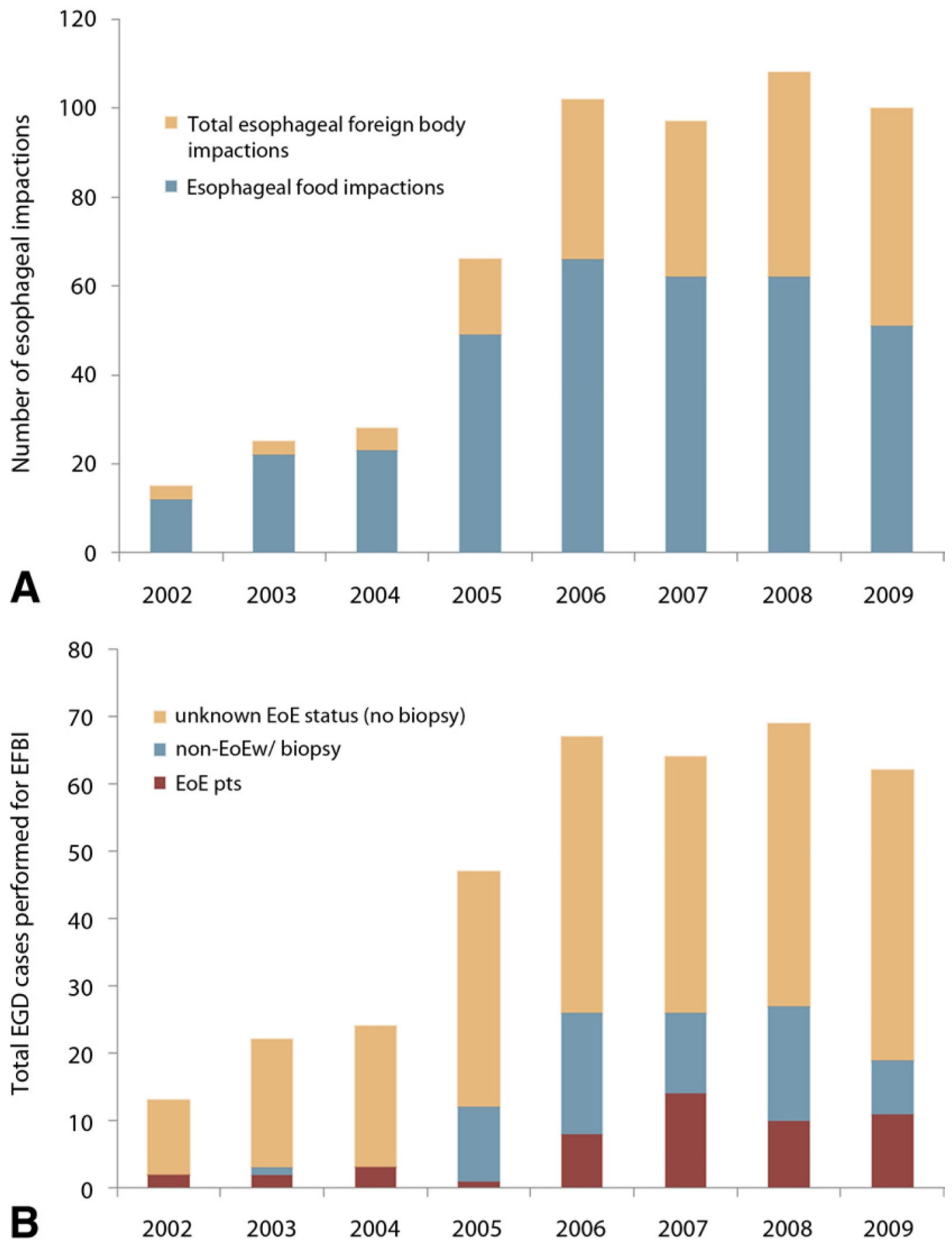

Figure 1.

A, Time trends of the number of esophageal foreign-body impactions (EFBIs) from 2002 to 2009 , divided between the total number of episodes (top of the blue bars) and the number of food impactions ( $\tan$ bars). B, Time trends of upper endoscopies performed for EFBI from 2002 to 2009, divided between confirmed eosinophilic esophagitis (EoE) patients (red bars), patients without EoE who had negative esophageal biopsy findings (blue bars), and patients with an unknown EoE status because they did not undergo esophageal biopsy (tan bars). 
TABLE 1

Clinical characteristics of patients with esophageal foreign-body impaction $(\mathrm{N}=548)$

\begin{tabular}{|lc|}
\hline & No. $(\%)$ \\
\hline Age, y, mean \pm SD & $37.3 \pm 30.2$ \\
\hline Sex & $224(41)$ \\
\hline Female & $324(59)$ \\
\hline Male & \\
\hline Race & $374(68)$ \\
\hline White & $111(20)$ \\
\hline Black & $28(5)$ \\
\hline Hispanic & $4(1)$ \\
\hline Asian & $2(<1)$ \\
\hline Native American & $29(5)$ \\
\hline Other & $114(21)$ \\
\hline Food impaction history & $137(25)$ \\
\hline History of food impaction & $73(13)$ \\
\hline Subsequent food impaction & $39(7)$ \\
\hline GI diagnoses & $264(48)$ \\
\hline EoE & $107(20)$ \\
\hline Diagnosed after EFBI episode & $45(8)$ \\
\hline Diagnosed before EFBI episode & $6(1)$ \\
\hline GERD/erosive esophagitis & $80(15)$ \\
\hline Esophageal stricture & $65(12)$ \\
\hline Hiatal hernia & $59(11)$ \\
\hline Schatzki's ring & $38(7)$ \\
\hline Achalasia & \\
\hline Cancer & \\
\hline Other ${ }^{*}$ & \\
\hline None & \\
\hline Impacted item ${ }^{\dagger}$ & \\
\hline Meat & \\
\hline Other food ${ }^{\ddagger}$ & \\
\hline Coin & \\
\hline Other & \\
\hline Unknown & \\
\hline
\end{tabular}

$S D$, Standard deviation; $E o E$, eosinophilic esophagitis; $E F B I$, esophageal foreign-body impaction.

* Other GI diagnoses include esophageal ulcer $(n=10)$, previous antireflux surgery $(n=23)$, Barrett's esophagus $(n=4)$, previous esophageal atresia surgery $(\mathrm{n}=2)$, previous tracheoesophageal fistula repair $(\mathrm{n}=10)$, other $(\mathrm{n}=58)$.

${ }^{\dagger}$ Impacted item as reported by patient or family description or by procedure report. 
${ }^{*}$ Examples of other food include beans, fruit, vegetable.

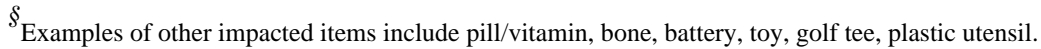


TABLE 2

Procedure characteristics for patients undergoing bolus extraction $(n=482)$

\begin{tabular}{|lc|}
\hline \multicolumn{2}{|c|}{ No. $(\%)$} \\
\hline Procedure type $^{*}$ & $363(75)$ \\
\hline EGD (performed by GI or surgical service) & $126(26)$ \\
\hline ENT laryngoscopy/esophagoscopy & $1(<1)$ \\
\hline Surgical exploration & \\
\hline Technique & $236(49)$ \\
\hline Extraction & $216(45)$ \\
\hline Advanced into stomach & $10(2)$ \\
\hline Lavage & $7(1)$ \\
\hline Aspiration & \\
\hline Tools used ${ }^{\dagger}$ & $116(24)$ \\
\hline Forceps (nonbiopsy) & $42(9)$ \\
\hline Biopsy forceps & $47(10)$ \\
\hline Roth net & $18(4)$ \\
\hline Snare & $27(6)$ \\
\hline Coin grasper & $12(2)$ \\
\hline Suction cap & $6(1)$ \\
\hline Other & $66(14)$ \\
\hline Underwent dilation at time of procedure & $98(27)$ \\
\hline Esophageal biopsy procured at time of procedure & $14(3)$ \\
\hline Complication during procedure/l & \\
\hline & \\
\hline
\end{tabular}

$E N T$, ear, nose, and throat.

Totals more than $100 \%$ because some patients had 2 procedures.

${ }^{\dagger}$ More than 1 tool was used for some patients.

* Other tools include catheter, clip, hemostat (used through rigid endoscope), and basket.

$\S_{\text {Of the }} 363$ patients who underwent EGD.

"Complication defined as unsuccessful endoscopic procedure for esophageal foreign-body impaction clearance, documented respiratory compromise, perforation, mucosal injury, cardiac arrhythmia, hypotension, or death. 
TABLE 3

Comparison of patients with food and nonfood impactions*

\begin{tabular}{|lccc|}
\hline & \multicolumn{3}{c|}{ Impaction type } \\
\cline { 2 - 4 } & $\begin{array}{l}\text { Food } \\
(\mathbf{n = ~ 3 4 7 )}\end{array}$ & $\begin{array}{l}\text { Nonfood } \\
(\mathbf{n}=\mathbf{1 9 5})\end{array}$ & $\boldsymbol{P}$ value \\
\hline Age, y, mean \pm SD & $50.4 \pm 25.3$ & $13.8 \pm 23.4$ & $<.001$ \\
\hline Female, no. (\%) & $134(39)$ & $85(44)$ & .24 \\
\hline White, no. (\%) & $267(77)$ & $102(53)$ & $<.001$ \\
\hline Multiple EFBI episodes, no. (\%) & $95(27 \%)$ & $6(3 \%)$ & $<.001$ \\
\hline Presumptive cause of EFBI, no. (\%) & & & \\
\hline EoE & $42(12)$ & $9(5) \dagger$ & .004 \\
\hline GERD & $48(14)$ & $8(4)$ & $<.001$ \\
\hline Other & $219(63)$ & $33(17)$ & $<.001$ \\
\hline Underwent procedure, no. (\%) & $301(87)$ & $176(91)$ & .39 \\
\hline Esophageal biopsy sample obtained, no. (\%) & $80(23)$ & $18(9)$ & $<.001$ \\
\hline
\end{tabular}

$S D$, Standard deviation; $E F B I$, esophageal foreign-body impaction; $E o E$, eosinophilic esophagitis.

Type of impaction was unclear in 6 patients. Those patients were excluded from this analysis. Means were compared with $t$ test, medians with Wilcoxon rank-sum, and proportions with $\chi^{2}$ test.

${ }^{\dagger}$ Of the 9 EoE patients with nonfood impaction, 1 had a coin impaction, 1 had a tooth impaction, and in the other 7 patients, the type of impacted nonfood item was not specified. 
TABLE 4

Comparison of EFBI patients with EoE and without a known diagnosis of EoE

\begin{tabular}{|lccc|}
\hline & $\begin{array}{c}\text { EoE } \\
(\mathbf{n = 5 1})\end{array}$ & $\begin{array}{c}\text { No EoE } \\
(\mathbf{n}=\mathbf{4 9 7})\end{array}$ & $\boldsymbol{P}$ value \\
\hline Age, y, mean \pm SD & $26.1 \pm 15.9$ & $38.5 \pm 31.1$ & .005 \\
\hline Female, no. (\%) & $9(18)$ & $215(43)$ & $<.001$ \\
\hline White, no. (\%) & $47(92)$ & $327(66)$ & $<.001$ \\
\hline Food impaction, no. (\%) & $42(82)$ & $305(61)$ & .007 \\
\hline Underwent procedure, no. $(\%)$ & $47(92)$ & $438(88)$ & .24 \\
\hline
\end{tabular}

$S D$, Standard deviation; $E F B I$, esophageal foreign-body impaction; $E o E$, eosinophilic esophagitis. 
TABLE 5

Comparison of single and multiple episodes of EFBI

\begin{tabular}{|lccc|}
\hline & $\begin{array}{c}\text { Single EFBI } \\
(\mathbf{n}=\mathbf{4 4 5})\end{array}$ & $\begin{array}{c}\text { Multiple } \\
\text { EFBIs } \\
(\mathbf{n = ~ 1 0 3})\end{array}$ & $\boldsymbol{P}$ value \\
\hline Age, y, mean \pm SD & $35.2 \pm 30.7$ & $46.5 \pm 26.4$ & $<.001$ \\
\hline Female, no. (\%) & $191(43)$ & $33(32)$ & .04 \\
\hline White, no. (\%) & $296(67)$ & $78(76)$ & .07 \\
\hline Diagnosis of GERD, no. (\%) & $34(8)$ & $23(22)$ & $<.001$ \\
\hline Diagnosis of EoE, no. (\%) & $33(7)$ & $18(17)$ & .002 \\
\hline Underwent procedure, no. (\%) & $388(87)$ & $94(91)$ & .27 \\
\hline
\end{tabular}

$S D$, Standard deviation; $E F B I$, esophageal foreign-body impaction; EoE, eosinophilic esophagitis.

Multiple EFBIs defined as 1 or more additional episode of EFBI, documented by multiple International Classification of Diseases, 9th Revision, Clinical Modification code matches and/or previous episodes of EFBI as described by the patient during the visit. 